\title{
Abductor Pollicis Longus
}

National Cancer Institute

\section{Source}

National Cancer Institute. Abductor Pollicis Longus. NCI Thesaurus. Code C52888.

A skeletal muscle of the forearm originating from the posterior surfaces of the ulna and radius and the interosseous membrane. 